Pacific Journal of Mathematic 


\title{
INVARIANT SUBSPACES OF A DIRECT SUM OF WEIGHTED SHIFTS
}

\author{
ERIC A. NordGren
}

\begin{abstract}
The invariant subspaces of a direct sum of finitely many copies of the adjoint of a monotone $1^{2}$ shift are shown to be spanned by the finite dimensional invariant subspaces that they include. For the case of two copies of such a shift, the invariant subspaces are characterized in terms of a spanning set of vectors, and all infinite dimensional invariant subspaces are shown to be cyclic.
\end{abstract}

It was shown by Donoghue [2] that if an operator $A$ on $H^{2}$ is defined by $A f(z)=z f(z / 2)$, then $A$ has a lattice of invariant subspaces anti-isomorphic to $\omega+1$. (This result has been generalized to a wider class of operators by Nikolskii [4].) Crimmins and Rosenthal [1] have shown that the direct product of two (or even countably many) lattices of invariant subspaces is attainable as the lattice of invariant subspaces of some operator. If $B$ and $C$ are operators on a separable Hilbert space such that their spectra are disjoint and no part of the spectrum of one is surrounded by spectrum of the other, then the lattice of invariant subspaces of $B \oplus C$ is the direct product of the lattice of $B$ by that of $C$. Thus, for example, their result gives the lattice of $(A+1) \oplus A$. This prompts the question: What is the lattice of $A \oplus A$ ? One answer is given by Nikolskii [5] in terms of operators in the commutant of $A \oplus A$. Actually, his results are valid for any operator that is a direct sum of a finite number of copies of a monotone $1^{p}$ shift (see [3], p. 97). Adjoints of such operators will be studied in this paper, and the following results will be derived. The invariant subspaces of such an adjoint are spanned by the finite dimensional invariant subspaces that they include, and these are invariant subspaces of finite dimensional nilpotent operators (Theorem 1 and Theorem 2, Corollary 2). The infinite dimensional invariant subspaces are cyclic except possibly for a finite dimensional summand (Theorem 2, Corollary 3 and Theorem 3, Corollary 3). For a sum of two copies of the adjoint of a monotone $1^{2}$ shift, the invariant subspaces can be completely characterized in terms of a spanning set of vectors (Theorem 1, Corollary 1).

We begin by establishing some notation. Although the natural setting for discussing shift operators is a sequence space, it will be somewhat more convenient to deal with functions on the unit circle $X$ in the complex plane $C$. Let $\mathscr{C}$ be a finite dimensional Hilbert 
space, $\mu$ normalized Lebesgue measure on $X$, and $\mathfrak{S}$ the Hilbert space of measurable norm square integrable functions from $X$ to $\mathscr{U}$ that are analytic. Thus, to say $F$ is in $\mathfrak{S}$ means $F$ is a measurable function from $X$ to $\mathscr{C}$ such that $\int\|F\|^{2} d \mu>\infty$, and if for each integer $n, e_{n}(z)=z^{n}$, then $\int F e_{n}^{*} d \mu=0$ whenever $n$ is negative. (The asterisk indicates the complex conjugate, and, as usual, functions that differ only on a set of measure zero are identified.) For each nonnegative integer $n$, define $w_{n}$ by $w_{n}=\int F e_{n}^{*} d \mu$ to obtain a sequence of coordinates of $F$ in $\mathscr{U}$, and then $F=\sum_{n=0}^{\infty} w_{n} e_{n}$.

A bounded sequence $\left\{\alpha_{0}, \alpha_{1}, \alpha_{2}, \cdots\right\}$ in $C$ induces a weighted shift operator $S^{*}$ on $\mathfrak{S}$ which is defined by

$$
S^{*} F=\sum_{n=0}^{\infty} \alpha_{n} w_{n} e_{n+1} .
$$

We will describe the invariant subspaces of the adjoint $S$ of such an operator for the case of a positive, monotonic and square summable weight sequence.

The connection between shifts as defined here and direct sums of shifts on $1^{2}$ is established in a standard manner. Choose an orthonormal basis $\left\{u_{1}, u_{2}, \cdots, u_{m}\right\}$ for $\mathscr{U}$. Then, if $F$ is in $\mathfrak{K}$, let $f_{j}(1 \leqq$ $j \leqq m$ ) be the sequence of Fourier coefficients with nonnegative index of ( $\mathrm{F}, u_{j}$ ), and identify $F$ with $f_{1} \oplus f_{2} \oplus \cdots \oplus f_{m}$. By this means, the shift of multiplicity $m$, defined by (1.1), is identified with $m$ copies of the shift on $1^{2}$ induced by the sequence $\left\{\alpha_{n}\right\}$.

2. Shifts of arbitrary finite multiplicity. For each nonnegative integer $n$, let $P_{n}$ be the projection of $\mathfrak{S}$ onto $\mathscr{C}$ that sends a vector into its $n^{\text {th }}$ coordinate; $P_{n} \sum_{j=0}^{\infty} w_{j} e_{j}=w_{n}$. Define subspaces $\Re_{n}$ of $\mathfrak{S}$ by $\Re_{n}=\left\{F:\right.$ if $j \geqq n$, then $\left.P_{j} F=0\right\}$, let $\Re_{\infty}$ be $\mathfrak{S}$ itself, and define the index of a vector in $\mathfrak{S}$ to be the smallest $n$ such that $\Re_{n}$ contains the vector. Consider a nontrivial invariant subspace $\mathfrak{M}$ of $S$, and let $N$ be the largest integer such that $\mathfrak{M}$ includes $\mathfrak{R}_{N}$. Let $\mathfrak{M}_{n}$ be $\mathfrak{M} \cap \mathfrak{\Omega}_{N+n+1}$, so $\mathfrak{M}_{n}$ consists of all vectors in $\mathfrak{M}$ having no nonzezo coordinate beyond the $N+n^{\text {th }}$. Finally, let $\mathscr{V}_{n}$ be $P_{N+n} \mathfrak{M}_{n}$. Then $\mathscr{V}_{n}$ is a subspace of $\mathscr{C}$, and the following assertion is easily verified.

Lemma 1. $\mathscr{U} \neq \mathscr{V}_{0} \supset \mathscr{Y}_{1} \supset \mathscr{V}_{2} \supset \cdots$.

It will be shown that every invariant subspace of $S$ is the span of the finite dimensional invariant subspaces that it includes (Theorem 2, Corollary). Theorem 1 below implies that every finite dimensional invariant subspace of $S$ is included in $K_{n}$ for some integer $n$. The 
restriction of $S$ to $\Re_{n}$ is a nilpotent operator of index $n$ on a space of dimension $m n$. Thus, all invariant subspaces of $S$ can be produced by forming spans of invariant subspaces of finite dimensional nilpotent operators.

THEOREM 1. Every invariant subspace of $S$ which is infinite dimensional or contains a vector of infinite index includes an infinite orthonormal set of vectors of finite index.

Proof. If $\mathfrak{M}$ is an invariant subspace of $S$ and $\mathfrak{M}$ contains vectors of arbitrarily large finite index, then the Gram Schmidt process may be used to complete the proof. Suppose therefore that $\mathfrak{M}$ contains a vector $F=\sum_{n=0}^{\infty} w_{n} e_{n}$ of infinite index. Induction will be used to establish the existence of an infinite orthonormal sequence $\left\{G_{0}, G_{1}, G_{2}, \cdots\right\}$ in $\mathfrak{M}$ such that the index of $G_{n}$ is no greater than $n+1$. Let $G_{-1}$ be 0 . Suppose an orthonormal sequence $\left\{G_{0}, G_{1}, \cdots, G_{m-1}\right\}$ has been found, which is in $\mathfrak{M}$, is empty if $m=0$, and has the asserted index property. Let $Q_{m}$ be the projection on $\Omega_{m}$, and let $R_{m}$ be the projection on the orthogonal complement of $\left\{G_{-1}, G_{0}, \cdots, G_{m-1}\right\}$. Choose a sequence of integers $n(k)$ such that (1) $\left\|w_{n(k)+m}\right\| \geqq\left\|w_{j}\right\|$ for all $j \geqq n(k)+m$, and (2) if $H_{n(k)}=Q_{m+1} R_{m} S^{n(k)} F$ then $\left\{H_{n(k)} /\left\|H_{n(k)}\right\|\right\}$ converges in the finite dimensional subspace $\Re_{m+1}$ to a unit vector $G_{m}$. Then $G_{m}$ is orthogonal to $\left\{G_{-1}, \cdots, G_{m+1}\right\}$ and of index no greater than $m+1$. The proof will be completed by showing that $G_{m}$ is in $\mathfrak{M}$.

Since $\left\{G_{0}, G_{1}, \cdots, G_{m-1}\right\}$ is included in $\Re_{m}$, it follows that the projection $R_{m}$ does not change the coefficient of $e_{m}$. Thus,

$$
\left\|H_{n(k)}\right\| \geqq \alpha_{m} \alpha_{m+1} \cdots \alpha_{m+n(k)-1}\left\|w_{n(k)+m}\right\| .
$$

The vector $R_{m} S^{n(k)} F$ is in $M$ for each $k$, and

$$
\begin{aligned}
& \|\| H_{n(k)}\left\|^{-1} R_{m} S^{n(k)} F-G_{m}\right\|^{2}=\|\| H_{n}(k)\left\|^{-1} H_{n(k)}-G_{m}\right\|^{2} \\
+ & \|\| H_{n(k)}\left\|^{-1} \sum_{j=m+1}^{\infty} \alpha_{j} \alpha_{j+1} \cdots a_{j+n(k)-1} w_{j+n(k)-1} e_{j}\right\|^{2} .
\end{aligned}
$$

By the definition of $G_{m}$, the first term on the right hand side of (2.2) converges to zero as $k$ tends to infinity. The inequality (2.1), the first condition on $n(k)$, and the hypothesis that $\left\{\alpha_{j}\right\}$ is monotonically decreasing, imply that the second term on the right hand side of (2.2) is no greater than $\alpha_{m}^{-2} \sum_{j=m+1}^{\infty} \alpha_{j+n(k)-1}^{2}$. This also converges to zero as $k$ tends to infinity, since $\left\{\alpha_{j}\right\}$ is square summable. Thus, $G_{m}$ is the limit of a sequence in $\mathfrak{M}$; hence, it is in $\mathfrak{M}$; and the theorem is proved.

REMARK. The proof above is a modification of a technique devised by S. Parrott to give an alternative proof of the result of Donoghue 
mentioned in the introduction. See [3], Problem 151.

From now on it will be assumed $\mathfrak{M}$ is an infinite dimensional invariant subspace of $S$. By Theorem 1 and Lemma 1 , if $\mathscr{V}^{-}=\bigcap_{n=0}^{\infty} \mathscr{V}_{n}^{*}$, then $\mathscr{V}^{-} \neq\{0\}$. The next task is to describe a convenient basis (not in general orthonormal) for $\mathfrak{M}_{n} \ominus \mathfrak{R}_{N}\left(=\mathfrak{M}_{n} \cap \mathfrak{N}_{N}^{\perp}\right)$, and it will suffice to do this for the special case in which $\mathscr{V}^{\wedge}=\mathscr{V}_{0}^{\wedge}$. Let $\left\{v_{1}, v_{2}, \cdots, v_{p}\right\}$ be an orthonormal basis for $\mathscr{V}$; and for each $j(1 \leqq j \leqq p)$, let $G_{j}$ be a vector of index $N+n+1$ in $\mathfrak{M}_{n}$ that has $v_{j}$ as leading coefficient, i.e.,

$$
G_{j}=v_{j} e_{N+n}+H_{j},
$$

where $H_{j}$ is a vector in $\Re_{N+n}$. The set $\left\{S^{n} G_{j}, S^{n-1} G_{j}, \cdots, G_{j}\right\}$ is included in $\mathfrak{M}_{n}$ for each $j$, and the projection of an appropriate multiple of $S^{n} G_{j}$ on the complement of $\Re_{N}$ is a vector $F_{j}(0)$ in $\mathfrak{M}$ such that

$$
F_{j}(0)=v_{j} e_{N} \cdot
$$

Suppose $\left\{F_{j}(0), F_{j}(1), \cdots, F_{j}(k-1)\right\}$ has been defined for $k \leqq n$ and for each $j$. Define $F_{j}(k)$ by

$$
\begin{aligned}
F_{j}(k) & =\left(\alpha_{N+n-1} \alpha_{N+n-2} \cdots \alpha_{N+k}\right)^{-1}\left[\left(1-Q_{N}\right) S^{n-k} G_{j}\right. \\
& \left.-\sum_{i=1}^{p} \sum_{m=0}^{k-1}\left(S^{n-k} G_{j}, v_{i} e_{N+m}\right) F_{i}(m)\right] .
\end{aligned}
$$

Thus $\mathrm{F}_{j}(k)$ is a vector of index $N+k+1$ in $\mathfrak{M} \ominus \Re_{N}$ such that its $N+k^{\text {th }}$ coordinate is $v_{j}$, and all its other coordinates are orthogonal to $\mathscr{Y}$; i.e.,

$$
F_{j}(k)=v_{j} e_{N+k}+\sum_{i=0}^{k-1} w(i, j, k) e_{N+i},
$$

where $w(i, j, k) \perp \mathscr{V}$ : The vectors $F_{j}(k)$ make up the desired basis. For each $j(1 \leqq j \leqq k)$ and each $k(k \geqq 1)$, let $u_{j}(k)=w(0, j, k)$. It will be shown that

$$
F_{j}(k)=v_{j} e_{N+k}+\sum_{i=0}^{k-1} \alpha(N, k, i) u_{j}(k-i) e_{N+i},
$$

where $\alpha(N, k, 0)=1$ and

$$
\alpha(N, k, i)=\left(\alpha_{N+k-1} \alpha_{N+k-2} \cdots \alpha_{N+k-i}\right) /\left(\alpha_{N} \alpha_{N+1} \cdots \alpha_{N+i-1}\right) .
$$

Since the $\mathrm{F}_{j}(k),(1 \leqq j \leqq p, 0 \leqq k \leqq n)$ form a basis for $\mathfrak{M}_{n} \theta \mathfrak{\Re}_{N}$,

$$
S F_{j}(k)=\alpha_{N+k-1} F_{j}(k-1)+\alpha_{N-1} u_{j}(k) e_{N-1} .
$$

It follows by induction, on equating coefficients of both sides of (2.5), that 


$$
\begin{aligned}
w(i, j, k) & =\alpha_{N+k-1} w(i-1, j, k-1) / \alpha_{N+i-1} \\
& =\alpha_{N+k-1} \alpha(N, k-1, i-1) u_{j}(k-i) / \alpha_{N+i-1} \\
& =\alpha(N, k, i) u_{j}(k-i) .
\end{aligned}
$$

For later use we insert here a fact concerning the coefficients $\alpha(N, k, i)$.

Lemma 2. $\sum_{i=1}^{k-1} \alpha(N, k, i)^{2} \leqq \alpha_{N+k-1}^{2} C_{N}$ for $k \geqq 2$, where $C_{N}=$ $\alpha_{N}^{-2}+\left(\alpha_{N} \alpha_{N+1}\right)^{-2} \sum_{i=N+1}^{\infty} \alpha_{i}^{2}$.

Proof. Define $c_{k}$ as the quotient of the term on the left hand side of the asserted inequality by $\left(\alpha_{N+k-1} / \alpha_{N}\right)^{2}$. We claim that $c_{k+1} \leqq$ $c_{k}+\left(\alpha_{N+k-1} / \alpha_{N+1}\right)^{2}$.

For,

$$
\begin{aligned}
c_{k+1} & -\left(\alpha_{N+k-1} / \alpha_{N+1}\right)^{2} \\
& =1+\sum_{\imath=j}^{k}\left(\alpha_{N+k-1} / \alpha_{N+i-1}\right)^{2}\left(\alpha_{N+k-2} \cdots \alpha_{N+k+1-i} / \alpha_{N+1} \cdots \alpha_{N+i-2}\right)^{2} \leqq c_{k} .
\end{aligned}
$$

Since $c_{2}=1$, it follows $c_{k} \leqq 1+\alpha_{N+1}^{-2} \sum_{i=N+1}^{\infty} \alpha_{i}^{2}$, and this implies the assertion.

THEOREM 2. Let $\mathscr{Y}$ be a nontrivial subspace of $\mathscr{U}$, let $\left\{v_{1} v_{2}, \cdots, v_{p}\right\}$ be an orthonormal basis for $\mathscr{Y} ;$ let $\left\{u_{j}(k)\right\}_{k=1}^{\infty}$ for $j=1,2, \cdots, p$ be norm square summable sequences in $\mathscr{V}^{\perp}$, and let $N$ be a nonnegative integer. Define $F_{j}(k)$ for $j=1,2, \cdots, p$ and $k=0,1,2, \cdots$ according to (2.3) and (2.4). Then the (closed) span $\mathfrak{M}$ of $\Re_{N}$ and all the vectors $F_{j}(k)$ is an invariant subspace of $S$ such that $P_{N+n}\left(\mathfrak{M} \cap \Omega_{N+n+1}\right)=\mathscr{V}^{-}$ for $n=0,1,2, \cdots$. Conversely, every invariant subspace $\mathfrak{M}$ of $S$ such that $P_{j}\left(\mathfrak{M} \cap \Re_{j+1}\right)$ is $\mathscr{U}$ for $j<N$ and $\mathscr{V}^{-}$for $j>N$ is obtained in this manner.

Proof. Given $F_{j}(k)$ as above, the relation (2.5) holds, and therefore the span $\mathfrak{M}$ of the $F_{j}(k)$ and $K_{N}$ is invariant under $S$. It will be shown that $P_{N+n}\left(\mathfrak{M} \cap \Re_{N+n+1}\right)=\mathscr{Y}^{-}$at the end of the proof.

Let $\mathfrak{M}$ be an invariant subspace of $S$ such that $P_{j}\left(\mathfrak{M} \cap K_{j+1}\right)$ is $\mathscr{U}$ if $\mathrm{j}<N$ and is a nontrivial subspace $\mathscr{Y}$ of $\mathscr{C}$ if $j \geqq N$. Then, as above, each $\mathfrak{M}_{n}$ is spanned by $\Re_{N}$ and vectors $F_{j}(k)$ for $1 \leqq j \leqq p$ and $0 \leqq k \leqq n$ which satisfy (2.3) or (2.4). It must be shown that the sequences $\left\{u_{j}(k)\right\}_{k=1}^{\infty}$ for $1 \leqq j \leqq p$ are norm square summable and that the $F_{j}(k)$ span $\mathfrak{M} \ominus \Re_{N}$.

First, it will be shown that each sequence $\left\{u_{j}(k)\right\}_{h=1}^{\infty}$ is bounded. If one of them, which we denote simply $\{u(k)\}_{k=1}^{\infty}$, is not bounded, then it has a subsequence $\left\{\left(u\left(k^{\prime}\right)\right\}\right.$ with the properties $\|u(i)\| \leqq\left\|u\left(k^{\prime}\right)\right\|$ if 
$i \leqq k^{\prime}$, and $\left\{u\left(k^{\prime}\right) /\left\|u\left(k^{\prime}\right)\right\|\right\}$ converges to a unit vector $u$ in $\mathscr{Y}^{\perp}$. Extending the convention of dropping subscripts to indicate all vectors with the same subscript as the unbounded sequence $\{u(k)\}$, we then have that $\left\|u\left(k^{\prime}\right)\right\|^{-1} F\left(k^{\prime}\right)$ is in $\mathfrak{M}$ for each $k$, and

$$
\begin{aligned}
\left\|u\left(k^{\prime}\right)\right\|^{-1} F\left(k^{\prime}\right)-u e_{N}=\left(\| u\left(k^{\prime}\right)^{-1} u\left(k^{\prime}\right)-u\right) e_{N} \\
+\sum_{i=1}^{k^{\prime}-1} \alpha\left(N, k^{\prime}, i\right)\left\|u\left(k^{\prime}\right)\right\|^{-1} u\left(k^{\prime}-i\right) e_{N+i}+\left\|u\left(k^{\prime}\right)\right\|^{-1} v e_{N+k^{\prime}} .
\end{aligned}
$$

As will be shown, the right hand side converges to zero as $k$ tends to infinity. It follows that $u e_{N}$ is in $\mathfrak{M}$ which is impossible since $u e_{N}$ is orthogonal to $\mathfrak{M}_{0}$, and this contradiction implies boundedness. To return to the above equation, note that the first term on the right hand side converges to zero by the choice of the subsequence, and the third term also does since $\left\{\left\|u\left(k^{\prime}\right)\right\|^{-1}\right\}$ converges to zero. As for the second term, the summands are orthogonal and of norm less than or equal $\alpha\left(N, k^{\prime}, i\right)$; therefore, the norm squared of the second term is no greater than $\sum_{i=1}^{k^{\prime}-1} \alpha\left(N, k^{\prime}, i\right)^{2}$, which tends to zero as $k$ tends to infinity by Lemma 2. The proof of boundedness is complete, so there is a constant $\beta$ such that $\left\|u_{j}(k)\right\| \leqq \beta$ for all $j$ and $k$.

Suppose next that one of the sequences $\left\{u_{j}(k)\right\}_{k=1}^{\infty}$ is not norm square summable, and denote this sequence $\{u(k)\}$. To derive a contratiction, the first step will be to produce a square summable sequence of complex numbers $\sigma_{k}$ together with a sequence of integers $n(j)$ such that the vectors $w_{j}$ in $\mathscr{V}^{\perp}$, defined by

$$
w_{j}=\sum_{k=j}^{n(j)} \sigma_{k} u(k)
$$

are all of norm at least one. This may be accomplished by taking an orthonormal basis $\left\{x_{1}, x_{2}, \cdots, x_{q}\right\}$ for $\mathscr{V}^{\perp}$ and considering the $q$ sequences $\left\{\left(u(k), x_{i}\right)\right\}_{k=1}^{\infty}$. By the Parsevaal identity at least one of these sequences, which we denote $\{(u(k)), x)\}$, is not square summable. Choose a square summable sequence $\left\{\sigma_{k}\right\}$ such that $\sigma_{k}(u(k), x) \geqq 0$ and $\sum_{k=1}^{\infty} \sigma_{k}(u(k), x)=\infty$ (see [3], p. 14), and corresponding to each $j$ choose $n(j)$ such that

$$
\sum_{k=j}^{n(j)} \sigma_{k}(u(k), x)>1
$$

With these choices,

$$
\left\|w_{j}\right\| \geqq\left|\left(\sum_{k=j}^{n(j)} \sigma_{k} u(k), x\right)\right|>1,
$$

and the first step is complete. Next, take a subsequence $\left\{w_{j^{\prime}}\right\}$ such that $\left\{w_{j^{\prime}} /\left\|w_{j^{\prime}}\right\|\right\}$ converges to a unit vector $w$ in $\mathscr{V}^{-1}$. The contradic- 
tion now arises because the sequence $\left\{\left\|w_{j^{\prime}}\right\|^{-1} \sum_{\substack{n\left(j^{\prime}\right) \\ k=j^{\prime}}} \sigma_{k} F(k)\right\}$ in $\mathfrak{M}$ converges to $w e_{N}$, which is orthogonal to $\mathfrak{M}_{0}$. (As above, $F(k)$ denotes that $F_{j}(k)$ with the same subscript as the sequence $\{u(k)\}$.) To see this, write $F(k)=v e_{N+k}+G(k)+u(k) e_{N}$, where

$$
G(k)=\sum_{i=1}^{k-1} \alpha(N, k, i) u(k-i) e_{N+i},
$$

and consider the difference

$$
\begin{aligned}
& \left\|w_{j^{\prime}}\right\|^{-1} \sum_{k=i^{\prime}}^{n\left(j^{\prime}\right)} \sigma_{k} F(k)-w e_{N}=\left\|w_{j^{\prime}}\right\|^{-1} \sum_{k=j^{\prime}}^{n\left(j^{\prime}\right)} \sigma_{k} v e_{N^{\prime}+k} \\
& \quad+\left\|w_{j^{\prime}}\right\|^{-1} \sum_{k=j^{\prime}}^{n\left(j^{\prime}\right)} \sigma_{k} G(k)+\left(\left\|w_{j^{\prime}}\right\|^{-1} \sum_{k=j^{\prime}}^{n\left(j^{\prime}\right)} \sigma_{k} u(k)-w\right) e_{N} \cdot
\end{aligned}
$$

On the right hand side of this equation, the first term tends to zero as $j$ tends to infinity because the sequence $\left\{\sigma_{k}\right\}$ is square summable, and the third term also does so by the choice of the subsequence $\left\{w_{j^{\prime}}\right\}$. By Lemma $2,\|G(k)\| \leqq \beta C_{N}^{1 / 2} \alpha_{N+k-1}$. Thus, by the triangle inequality and the fact that $\left\|w_{j^{\prime}}\right\|^{-1}<1$, the second term is no greater in norm than $\beta C_{N}^{1 / 2} \sum_{\substack{n\left(j^{\prime}\right) \\ k=j^{\prime}}}\left|\sigma_{k}\right| \alpha_{N+k-1}$, which tends to zero as $j$ tends to infinity since both $\left\{\sigma_{k}\right\}$ and $\left\{\alpha_{k}\right\}$ are square summable. Hence each of the sequences $\left\{u_{j}(k)\right\}_{k=1}^{\infty}$ is norm square summable.

To complete the proof we will need the fact that for each $j$, $\left\{\sigma_{k} F_{j}(k)\right\}_{k=0}^{\infty}$ is summable in $\mathfrak{g}$ whenever $\left\{\sigma_{k}\right\}$ is a square summable sequence of complex numbers. If $m$ and $n$ are integers, then, dropping the subscript $j$ and using the notation introduced in the preceding paragraph, we may write

$$
\sum_{k=m}^{n} \sigma_{k} F(k)=\sum_{k=m}^{n} \sigma_{k} v e_{N+k}+\sum_{k=m}^{n} \sigma_{k} G(k)+\sum_{k=m}^{n} \sigma_{k} u(k) e_{N} \cdot
$$

As above, the first two terms on the right hand side can be made small by taking $m$ and $n$ sufficiently large, but in addition the third term has norm no greater than $\sum_{k=m}^{u}\left|\sigma_{k}\right|\|u(k)\|$, which can also be made small by taking $m$ and $n$ large since $\{\|u(k)\|\}$ is now also known to be square summable. This implies that $\left\{\sigma_{k} F_{j}(k)\right\}_{k=0}^{\infty}$ is summable in $\mathfrak{S}$.

To see that the $F_{j}(k)$ span $\mathfrak{M} \ominus \Re_{N}$ suppose $F$ in $\mathfrak{M}$ is orthogonal to $\Re_{N}$; define $\sigma_{j}(k)$ by $\sigma_{j}(k)=\left(F, v_{j} e_{N+k}\right)$ for $k=0,1,2, \cdots$ and $j=$ $1,2, \cdots, p$; and define $G$ by $G=\sum_{j=1}^{p} \sum_{k=0}^{\infty} \sigma_{j}(k) F_{j}(k)$. By the remarks of the preceding paragraph the definition of $G$ is permissible, and thus $F-G$ is a vector in $\mathfrak{M} \ominus \Re_{N}$ such that $P_{N+n}(F-G)$ is orthogonal to $\mathscr{V}^{-}$for all $n$. If $F-G$ were not zero, then the technique of Theorem 1 could be employed to produce a vector in $\mathfrak{M}_{0}$ orthogonal to $\left\{F_{1}(0)\right.$, $\left.F_{2}(0), \cdots, F_{p}(0)\right\}$, which is impossible. Thus $F=G$ and the vectors $F_{j}(k)$ span $\mathfrak{M} \ominus \Re_{N}$. 
The final step is to supply the proof that if $\mathfrak{M}$ is the span of $\mathscr{\Omega}_{N}$ and the vectors $F_{j}(k)$, then $P_{N+n}\left(\mathfrak{M} \cap \Re_{N+n+1}\right)=\mathscr{Y}^{-}$for $n=0,1,2, \cdots$. That the set on the left includes the one on the right is clear. To obtain the opposite inclusion, by Lemma 1, it will suffice to prove that if $w$ is any nonzero vector in $\mathscr{V}^{-\perp}$, then $w e_{N}$ is at a positive distance from the span of the $F_{j}(k)$. For each $j(1 \leqq j \leqq p)$, let $\left\{\sigma_{j}(k)\right\}_{k=0}^{\infty}$ be any eventually null sequence of complex numbers. Then

$$
\begin{aligned}
\| w e_{N} & -\sum_{j=1}^{p} \sum_{k=0}^{\infty} \sigma_{j}(k) F_{j}(k)\left\|^{2} \geqq\right\| \sum_{j=1}^{n} \sum_{k=0}^{\infty} \sigma_{j}(k) v_{j} e_{N+k} \|^{2} \\
& +\left\|\left(w-\sum_{j=1}^{p} \sum_{k=1}^{\infty} \sigma_{j}(k) u_{j}(k)\right) e_{N}\right\|^{2} \\
& =\sum_{j=1}^{p} \sum_{k=0}^{\infty}\left|\sigma_{j}(k)\right|^{2}+\left\|w-\sum_{j=1}^{p} \sum_{k=1}^{\infty} \sigma_{j}(k) u_{j}(k)\right\|^{2},
\end{aligned}
$$

and the sum on the right may be shown to be bounded away from zero independently of the choice of the $\sigma_{j}(k)$. This completes the proof of the theorem.

COROLlaRy 1. If $\mathscr{W}$ has dimension two, then every nontrivial invariant subspace of $S$ is either finite dimensional or else consists of the span of $\Omega_{N}$ for some $N(\geqq 0)$ and a sequence $\left\{F_{n}\right\}_{n=0}^{\infty}$ in which each $F_{n}$ is of index $N+n+1$. The vectors $F_{n}$ may be defined by means of an orthonormal basis $\{v, u\}$ for $\mathscr{U}$ and a square summable sequence $\left\{\rho_{k}\right\}_{k=1}^{\infty}$ in $C: F_{0}=v e_{N}$; and if $n>0$, then

$$
F_{n}=v e_{N+n}+\sum_{j=0}^{n-1} \alpha(N, n, j) \rho_{n-j} u e_{N+j} .
$$

REMARK. A complete description of the finite dimensional invariant subspaces of $S$ in the above terms may also be given in this case. For a finite dimensional invariant subspace, the sequence $\left\{F_{n}\right\}$ is merely finite or nonexistent.

Proof. If $\mathscr{C}$ has dimension two, then every nontrivial infinite dimensional invariant subspace of $S$ satisfies the conditions of the theorem with $p=1$.

CoROllary 2. Every invariant subspace of $S$ is spanned by the finite dimensional ones which it includes, and each of these consists of vectors of finite index.

Proof. Since $\mathfrak{S}$ itself is spanned by the finite dimensional invariant subspaces $\Re_{n}$, it is sufficient to consider the case of a nontrivial infinite dimensional invariant subspace $\mathfrak{M}$ of $S$. Define the sequence of sub- 
spaces $\mathscr{V}_{n}^{-}$of $\mathscr{C}$ as in Lemma 1 . In general the intersection $\mathscr{V}^{-}$of these subspaces will be smaller than $\mathscr{V}_{0}^{0}$, but Lemma 1 and Theorem 1 imply it will be nontrivial. Let $\mathscr{V}_{q}$ be the first subspace in the sequence which is equal to $\mathscr{V}$. If $M=N+q$, then define $\mathfrak{N}$ as $\mathfrak{M}+\mathfrak{\Omega}_{M}$ to obtain a (closed) invariant subspace of $S$ which satisfies the conditions of Theorem 2. Clearly,

$$
\mathfrak{R}=\mathfrak{M}+\left(\mathfrak{R}_{M} \ominus \mathfrak{M}_{q-1}\right),
$$

and this is a direct sum decomposition of $\mathfrak{R}$. Since $\Re_{M} \ominus \mathfrak{M}_{q-1}$ is finite dimensional, the projection of $\mathfrak{N}$ onto $\mathfrak{M}$ along $\mathfrak{R}_{M} \ominus \mathfrak{M}_{q-1}$ is continuous. Thus, if $F$ is in $\mathfrak{M}$, then it is the limit of a sequence of vectors of finite index in $\mathfrak{R}$. The image of this sequence under the projection on $\mathfrak{M}$ is a sequence of vectors of finite index in $\mathfrak{M}$, and it also converges to $\mathrm{F}$. This proves the corollary.

COROLlaRY 3. Every invariant subspace of $S$ is the sum of a cyclic subspace and a finite dimensional invariant subspace of $S$.

Proof. It may be shown that $\mathfrak{S}$ itself is cyclic (see [3], p. 282, for an analogous situation). Suppose $\mathfrak{M}$ is an invariant subspace of $S$ that has the form required for an application of Theorem 2, and let $F_{j}(k)$ be the set of vectors that spans $\mathfrak{M} \ominus \Re_{N}$. Define $F$ by

$$
F=\sum_{k=0}^{\infty} \sum_{j=1}^{p}(p k+j)^{-1} F_{j}(p k+j),
$$

and consider the sum $\mathfrak{M}^{\prime}$ of the cyclic subspace generated by $F$ and the finite dimensional invariant subspace $\Omega_{N}$. Since the projection of $S_{n} F_{j}(k)$ on the orthogonal complement of $\mathfrak{M}_{N}$ is

$$
\alpha_{N+K-1} \cdots \alpha_{N+K-1} F_{j}(k-n)
$$

if $k \geqq n$, an induction argument may be used to show that $\mathfrak{M}^{\prime}$ contains all the $F_{j}(k)$, and thus $\mathfrak{M}^{\prime}$ includes $\mathfrak{M}$. The opposite inclusion is trivial, and the proof for the special case is complete.

If $\mathfrak{M}$ is an arbitary nontrivial infinite dimensional invariant subspace of $S$, then define $\mathfrak{N}$ as in the proof of the preceding corollary. Take a vector $F$ in $\mathfrak{N}$ such that the sum of the cyclic subspace it generates and $\Re_{M}$ is $\mathfrak{N}$. There is a vector $G$ in $\mathfrak{M}$ such that the difference $F-G$ has index at most $\mathfrak{M}$. Consider the sum $\mathfrak{M}^{\prime}$ of the cyclic subspace generated by $G$ and the finite dimensional invariant subspace $\mathfrak{M}_{q-1}$. It is clear that $\mathfrak{M}^{\prime}$ is included in $\mathfrak{M}$. If $H$ is in $\mathfrak{M}$, then $H=F_{1}+F_{2}$, where $F_{1}$ is in the cyclic subspace generated by $F_{1}$ and $F_{2}$ is in $\Omega_{M}$. Further, $F_{1}=G_{1}+G_{2}$, where $G_{1}$ is in the cyclic subspace generated by $G$, and $G_{2}$ is in $\Re_{M}$. Then $H-G_{1}=G_{2}+F_{2}$ 
is in $\mathfrak{M} \cap \mathfrak{R}_{M}$, i.e., in $\mathfrak{M}_{q-1}$, and it follows that $H$ is in $\mathfrak{M}^{\prime}$. This establishes that $\mathfrak{M}$ is included in $\mathfrak{M}^{\prime}$, which completes the proof.

REMARK. In case $\mathscr{C}$ is two dimensional every infinite dimensional invariant subspace of $S$ is cyclic (Theorem 3, Corollary 3). This is not true for higher dimensions, as may be seen by considering the case in which $\mathscr{C} C$ is three dimensional and the invariant subspace is the sum of $\Re_{1}$ and a slice through a one-dimensional subspace of $\mathscr{C}$.

3. Shifts of multiplicity 2 . In the special case under consideration a complete characterization of the invariant subspaces of $S$ has been obtained (Theorem 2, Corollary 1). An infinite dimensional invariant subspace $\mathfrak{M}\left(N, v, u,\left\{\rho_{k}\right\}\right)$ is determined by a nonnegative integer $N$, an orthonormal basis $\{v, u\}$ for $\mathscr{C}$, and a square summable sequence $\left\{\rho_{k}\right\}$ in $C$. It is easy to see that $\mathfrak{M}\left(N, v, u,\left\{\rho_{k}\right\}\right)=M\left(N^{\prime}, v^{\prime}, u^{\prime},\left\{\rho^{\prime}{ }_{k}\right\}\right)$ if $N=N^{\prime}$ and there exist complex constants $\alpha$ and $\beta$ of modulus one such that $v=\alpha v^{\prime}, u=\beta u^{\prime}$ and $\rho_{k}=\alpha \beta^{*} \rho^{\prime}{ }_{k}$. The converse of this statement is contained in the following theorem.

Theorem 3. If $\{v, u\}$ and $\left\{v^{\prime}, u^{\prime}\right\}$ are bases for $\mathscr{U}$, and if $\left\{\rho_{k}\right\}$ and $\left\{\sigma_{k}\right\}$ are square summable sequences in $C$, then

$$
\mathfrak{M}\left(M, v, u,\left\{\boldsymbol{\theta}_{k}\right\}\right) \subset \mathfrak{M}\left(N, v^{\prime}, u^{\prime},\left\{\sigma_{k}\right\}\right)
$$

if and only if

(1) $M \leqq N$,

(2) there exist constants $\alpha$ and $\beta$ of unit modulus such that $v=\alpha v^{\prime}, u=\beta u^{\prime}$ and

(3) $\rho_{k}=\sigma_{k} \alpha(M, N-M+k, N-M)^{-1} \alpha \beta^{*}$. The inclusion is proper if and only if $M<N$.

Proof. Suppose the three conditions are satisfied for invariant subspaces $\mathfrak{M}$ and $\mathfrak{R}$, where $\mathfrak{M}=\mathfrak{M}\left(M, v, u,\left\{\rho_{k}\right\}\right)$ and $\mathfrak{N}=\mathfrak{M}\left(N, v^{\prime}, u^{\prime}\right.$, $\left.\left\{\sigma_{k}\right\}\right)$. Let $F_{n}$ be the sequence in $\mathfrak{M}$ determined by $v, u$ and the sequence $\left\{\rho_{k}\right\}$, and let $\left\{G_{n}\right\}$ be the analogous sequence in $\mathfrak{R}$. Since condition (1) implies that $\Omega_{M}$ is included in $\mathfrak{R}$, it suffices to show that $F_{n}$ is in $\mathfrak{N}$ for each $n$. This is immediate if $\mathrm{n} \leqq N-M$, for then $F_{n}$ is in the span of $\Re_{N}$ and $v^{\prime} e_{N}$. If $k>0$, then a calculation using the third assumption shows that

$$
\alpha(M, N-M+k, N-M+j) \rho_{k-j}=\alpha(N, k, j) \sigma_{k-j} \alpha \beta^{*}
$$

for each $j(0 \leqq j \leqq k)$, and it follows from this that

$$
F_{N-M+k}=\alpha G_{k}+H_{k}
$$


where $H_{k}$ is a vector in $\Re_{\Lambda}$. Thus $\mathfrak{M}$ is included in $\mathfrak{R}$.

Conversely, suppose $\mathfrak{M}$ and $\mathfrak{N}$ are two invariant subspaces of $S$, as in the preceding paragraph, such that $\mathfrak{N}$ includes $\mathfrak{M}$. Trivially, $\mathfrak{R}_{M}$ is included in $\mathfrak{R}$, and this implies $M \leqq N$. Since $F_{N-M}=v e_{N^{-}}+H_{1}$, where $H_{1}$ is in $\Omega_{N}$, it follows $v e_{N^{N}}=\alpha G_{0}=\alpha v^{\prime} e_{N}$, where $\alpha$ is a complex number of unit modulus. Hence, $v=\alpha v^{\prime}$. Since

$$
F_{N-M+1}=v e_{N+1}+\alpha(M, N-M+1, N-M) \rho_{1} u e_{N}+H_{2},
$$

where $H_{2}$ is a vector in $\Re_{N}$, it follows that

$$
F_{N-M+1}-H_{2}=\alpha G_{1}=\alpha v^{\prime} e_{N+1}+\alpha \sigma_{1} u^{\prime} e_{N},
$$

and hence, $u=\beta u^{\prime}$ for some $\beta$ of unit modulus, and

$$
\rho_{1}=\sigma_{1} \alpha(M, N-M+1, N-M)^{-1} \alpha \beta^{*} .
$$

Similarly, $F_{N-I I+k}$ is in $\mathfrak{N}$; its projection on the orthogonal complement of $\Omega_{N}$ is $\alpha G_{k}$; and a comparison of the coefficient of $e_{N}$ in this projection with the corresponding coefficient in $G_{k}$ yields the third condition.

Finally, it is clear that the inclusion is proper if $M<N$. If $\mathfrak{M}$ is included in $\mathfrak{R}$ and $M=N$, then since $\mathfrak{R} \cap \Re_{N+k}$ has dimension $2 N+k$, and includes $\left\{F_{0}, F_{1}, \cdots, F_{k-1}\right\}$ and $\mathfrak{\Re}_{N}$, it follows that $\mathfrak{N} \cap \mathfrak{\Re}_{N+k}=\mathfrak{M} \cap \mathfrak{R}_{N+k}$. Hence $\mathfrak{M}=\mathfrak{N}$ and the theorem is proved.

Corollary 1. An infinite dimensional invariant subspace $\mathfrak{M}\left(N, v, u,\left\{\sigma_{k}\right\}\right)$ of $S$ properly includes another infinite dimensional invariant subspace of $S$ if and only if $N>0$ and $\left.\sum_{k=1}^{\infty}\left|\sigma_{k}\right| \alpha_{N+k-1}\right|^{2}<\infty$.

Proof. If the condition holds, then $\mathfrak{M}\left(N-1, u, v,\left\{\alpha_{N-1} \sigma_{k} / \alpha_{N+k-1}\right\}\right)$ is an invariant subspace of $S$ which is properly included in the given one. Conversely, if $\mathfrak{M}\left(M, v, u,\left\{\rho_{k}\right\}\right)$ is properly included in $\mathfrak{M}(N, v, u$, $\left.\left\{\sigma_{k}\right\}\right)$, then $0 \leqq M<N$, and

$$
\left|\alpha_{M} \sigma_{k} / \alpha_{N+k-1}\right| \leqq \alpha(M, N-M+k, N-M)^{-1}\left|\sigma_{k}\right|=\left|\rho_{k}\right| \cdot
$$

Hence, square summability of $\left\{\sigma_{k} / \alpha_{N+k-1}\right\}$ follows from that of $\left\{\rho_{k}\right\}$, which completes the proof.

CoRollary 2. Every infinite dimensional invariant subspace of $S$ includes at most finitely many infinite dimensional invariant subspaces of $S$, and these are linearly ordered.

Proof. This follows directly from the theorem and preceding corollary. 
COROLlary 3. Every infinite dimensional invariant subspace of $S$ is cyclic.

Proof. Let $\Re$ be an infinite dimensional invariant subspace of $S$. The case of $\mathfrak{R}$ trivial was considered in Corollary 3 of Theorem 2, so we suppose $\mathfrak{N}=\mathfrak{M}(N, v, u,\{\sigma\})$. Let $\mathfrak{M}$ be the unique minimal infinite dimensional invariant subspace of $S$ included in $\mathfrak{R}$, and let $F$ be a vector of infinite index in $\mathfrak{M}$. If $\mathfrak{N}=\mathfrak{M}$, then $F$ is cyclic for $\mathfrak{N}$, and we are done. If $\mathfrak{M}$ is properly included in $\mathfrak{R}$, then define $G$ by $G=F+v e_{N-1}$, and let $\mathfrak{N}^{\prime}$ be the cyclic subspace determined by $G$. Since $\mathfrak{N}^{\prime}$ is included in $\mathfrak{N}$ and since $\mathfrak{M}$ is the unique minimal infinite dimensional invariant subspace of $S$ included in $\mathfrak{R}$, it follows that $\mathfrak{N}^{\prime}$ includes $\mathfrak{M}$. Thus $F$ is in $\mathfrak{R}^{\prime} ; v e_{N-1}$ is in $\mathfrak{N}^{\prime}$; and it follows easily that $\mathfrak{R}_{N}$ is included in $\mathfrak{N}^{\prime}$. But this implies that $\mathfrak{N}=\mathfrak{N}^{\prime}$, and hence $\mathfrak{N}$ is cyclic.

Remarks. 1. The dimension condition in Corollary 3 is clearly necessary since $\mathscr{R}_{1}$, for example, is not cyclic.

2. Throughout this paper it has been assumed that the sequence $\left\{\alpha_{n}\right\}$ which determines $S$ is monotonically decreasing and square summable. In fact, it is possible to get by with a somewhat weaker hopothesis. If the sequence $\left\{\alpha_{n}\right\}$ consists of positive terms, is eventually monotonically decreasing and belongs to some $1^{p}$ class $(0<p<\infty)$, then all the above proofs may be modified to yield the same results.

\section{REFERENCES}

1. T. Crimmins and P. Rosenthal, On the decomposition of invariant subspaces, Bull. Amer. Math. Soc. 73 (1967), 97-99.

2. W. F. Donoghue, The lattice of invariant subspaces of a completely continuous quasinilpotent transformation, Pacific J. Math. 7 (1957), 1031-1035.

3. P. R. Halmos, A Hilbert Space problem Book, Van Nostrand, Princeton, New Jersey, 1967.

4. N. K. Nikolskii, Invariant subspaces of certain completely continuous operators, Vestnik Leningrad University 7 (1965), 68-77 (Russian).

5. — The unicellularity and nonunicellularity of weighted shift operators, Dokl. Akad. Nauk SSSR (2) 172 (1967), Soviet Math. Dokl 8 (1967), 91-94.

Received August 28, 1967.

UNIVERSITY OF NEW HAMPSHIRE 


\section{PACIFIC JOURNAL OF MATHEMATICS}

\section{EDITORS}

\section{H. ROYDEN}

Stanford University

Stanford, California

\section{J. Dugundu}

Department of Mathematics University of Southern California Los Angeles, California 90007

RICHARD ARENS

University of California Los Angeles, California 90024

ASSOCIATE EDITORS
E. F. Beckenbach
B. H. NEUMANN
F. WoLF
K. YosidA

\section{SUPPORTING INSTITUTIONS}

\author{
UNIVERSITY OF BRITISH COLUMBIA \\ CALIFORNIA INSTITUTE OF TECHNOLOGY \\ UNIVERSITY OF CALIFORNIA \\ MONTANA STATE UNIVERSITY \\ UNIVERSITY OF NEVADA \\ NEW MEXICO STATE UNIVERSITY \\ OREGON STATE UNIVERSITY \\ UNIVERSITY OF OREGON \\ OSAKA UNIVERSITY \\ UNIVERSITY OF SOUTHERN CALIFORNIA
}

STANFORD UNIVERSITY

UNIVERSITY OF TOKYO

UNIVERSITY OF UTAH

WASHINGTON STATE UNIVERSITY

UNIVERSITY OF WASHINGTON

AMERICAN MATHEMATICAL SOCIETY
CHEVRON RESEARCH CORPORATION
TRW SYSTEMS

AMERICAN MATHEMATICAL SOCIETY

TRW SYSTEMS

NAVAL WEAPONS CENTER

Mathematical papers intended for publication in the Pacific Journal of Mathematics should be in typed form or offset-reproduced, double spaced with large margins. Underline Greek letters in red, German in green, and script in blue. The first paragraph or two must be capable of being used separately as a synopsis of the entire paper. It should not contain references to the bibliography. Manuscripts, in duplicate if possible, may be sent to any one of the four editors. All other communications to the editors should be addressed to the managing editor, Richard Arens, University of California, Los Angeles, California 90024.

Each author of each article receives 50 reprints free of charge; additional copies may be obtained at cost in multiples of 50 .

The Pacific Journal of Mathematics is published monthly. Effective with Volume 16 the price per volume (3 numbers) is $\$ 8.00$; single issues, $\$ 3.00$. Special price for current issues to individual faculty members of supporting institutions and to individual members of the American Mathematical Society: $\$ 4.00$ per volume; single issues $\$ 1.50$. Back numbers are available.

Subscriptions, orders for back numbers, and changes of address should be sent to Pacific Journal of Mathematics, 103 Highland Boulevard, Berkeley 8, California.

Printed at Kokusai Bunken Insatsusha (International Academic Printing Co., Ltd.), 7-17, Fujimi 2-chome, Chiyoda-ku, Tokyo, Japan.

PUBLISHED BY PACIFIC JOURNAL OF MATHEMATICS, A NON-PROFIT CORPORATION

The Supporting Institutions listed above contribute to the cost of publication of this Journal, but they are not owners of publishers and have no responsibility for its content or policies. 


\section{Pacific Journal of Mathematics \\ Vol. 27, No. 3}

March, 1968

Charles A. Akemann, Invariant subspaces of $C(G) \ldots \ldots \ldots \ldots \ldots \ldots . \ldots 41$

Dan Amir and Zvi Ziegler, Generalized convexity cones and their duals ... . 425

Raymond Balbes, On ( $J, M, \mathrm{~m})$-extensions of order sums of distributive

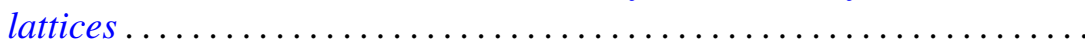

Jan-Erik Björk, Extensions of the maximal ideal space of a function algebra ........................................ 453

Frank Castagna, Sums of automorphisms of a primary abelian group ...... 463

Theodore Seio Chihara, On determinate Hamburger moment problems ..... .

Zeev Ditzian, Convolution transforms whose inversion function has complex

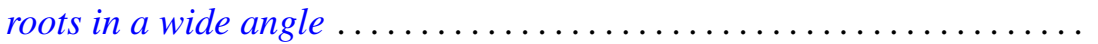

Myron Goldstein, On a paper of Rao .

Velmer B. Headley and Charles Andrew Swanson, Oscillation criteria for

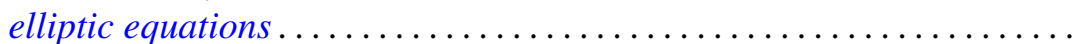

John Willard Heidel, Qualitative behavior of solutions of a third order nonlinear differential equation............................

Alan Carleton Hindmarsh, Pick's conditions and analyticity.............

Bruce Ansgar Jensen and Donald Wright Miller, Commutative semigroups

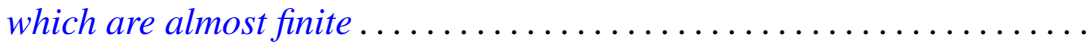

Lynn Clifford Kurtz and Don Harrell Tucker, An extended form of the

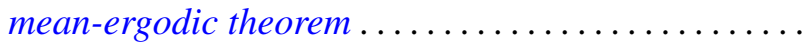

S. P. Lloyd, Feller boundary induced by a transition operator ...

Henry B. Mann, Josephine Mitchell and Lowell Schoenfeld, A new proof of the maximum principle for doubly-harmonic functions ...

Robert Einsohn Mosher, The product formula for the third obstruction ..

Sam Bernard Nadler, Jr., Sequences of contractions and fixed points ...

Eric Albert Nordgren, Invariant subspaces of a direct sum of weighted shifts...

Fred Richman, Thin abelian p-groups ...

Jordan Tobias Rosenbaum, Simultaneous interpolation in $\mathrm{H}_{2}$. II ...

Charles Thomas Scarborough, Minimal Urysohn spaces .

Malcolm Jay Sherman, Disjoint invariant subspaces..... .

Joel John Westman, Harmonic analysis on groupoids....

621

William Jennings Wickless, Quasi-isomorphism and TFM

Minoru Hasegawa, Correction to "On the convergence of resolvents of operators" 